\title{
Blocking the BK channel impedes acquisition of trace eyeblink conditioning
}

\author{
Elizabeth A. Matthews ${ }^{1}$ and John F. Disterhoft \\ Department of Physiology, Northwestern University, Chicago, Illinois 60611, USA
}

\begin{abstract}
Big- $\mathrm{K}^{+}$conductance (BK)-channel mediated fast afterhyperpolarizations (AHPs) following action potentials are reduced after eyeblink conditioning. Blocking BK channels with paxilline increases evoked firing frequency in vitro and spontaneous pyramidal activity in vivo. To examine how increased excitability after BK-channel blockade affects learning, rats received bilateral infusions of paxilline, saline, or nothing into hippocampal CAl prior to trace eyeblink conditioning. The drug group was slower to acquire the task, but learning was not completely impaired. This suggests that nonspecific increases in excitability and baseline neuronal firing rates caused by in vivo blockade of the BK channel may disrupt correct processing of inputs, thereby impairing hippocampus-dependent learning.
\end{abstract}

Learning and increased neuronal intrinsic excitability are strongly correlated, although a causal relationship has not yet been definitively established (Disterhoft and Oh 2006). One of the mechanisms of increased excitability is through reduction of potassium currents, which cause afterhyperpolarizations (AHP). Afterhyperpolarizations in pyramidal cells can be divided into three categories based upon their timing and duration. The fast AHP lasts only 2-5 ms, follows the depolarizing phase of individual action potentials, and is mediated largely by the big- $\mathrm{K}^{+}$ conductance (BK) channel. The post-burst AHP has a medium (50$100 \mathrm{~ms}$ ) and a slow (1-2 s) component, and follows trains or bursts of action potentials. The medium AHP is carried by apamin sensitive small- $\mathrm{K}^{+}$conductance (SK) channels, but the channel(s), which carry the slow AHP, are still unknown (Storm 1987; Disterhoft and Oh 2006).

Learning-related reductions in the post-burst AHP are well documented (for review see Disterhoft and Oh 2006). Additionally, pharmacological modulators of the post-burst AHP alter learning in an expected manner-compounds that reduce the AHP improve learning (galantamine [Simon et al. 2004] and nimodopine [Deyo et al. 1989]). Learning-related reductions of the fast AHP are also seen in prefrontal cortex pyramidal neurons after extinction of fear conditioning (Santini et al. 2008) and in CA1 hippocampal pyramidal neurons after learning trace eyeblink conditioning (tEBC) (Matthews et al. 2008). In vitro whole-cell recordings show that blocking the BK channel with either paxilline or iberiotoxin increases the firing rate to a step current injection (Nelson et al. 2003). Likewise, injection of paxilline into the hippocampus increases the in vivo spontaneous firing frequency of hippocampal CA1 neurons of awake freely moving rats up to 2.5-fold over saline injections (Matthews et al. 2008), indicating that the BK-mediated fast AHP plays an important role in intrinsic excitability. The current study was undertaken to determine whether pharmacologically reducing the fast AHP during training would improve trace eyeblink conditioning.

Experimental subjects were 3- to 4-mo old Fisher $344 \mathrm{X}$ Brown Norway F1-hybrid rats. Animals were housed in pairs, in a climate-controlled facility with a 12:12 light-dark cycle, and ad libitum access to food and water. Procedures were in accordance

\footnotetext{
'Corresponding author.

E-mail elizabethmatthews2009@u.northwestern.edu; fax (312) 503-3263.

Article is online at http://www.learnmem.org/cgi/doi/10.1101/lm.1289809.
}

with the guidelines of the Northwestern University Animal Care and Use Committee and conformed to NIH standards (NIH Publication No. 80-23). All efforts were made to minimize the number of animals utilized and their discomfort. Thirty-seven rats were originally included in the study, however 13 were excluded from the experiment due to incorrect cannulae placement, faulty EMG signal, or unrelated health issues. The final groups included in the study were nine drug animals, seven vehicle animals, eight sham animals, and six non-cannulated animals.

Guide cannulae (26 gauge stainless steel) were bilaterally implanted at $-3.6 \mathrm{~mm} \mathrm{AP}, \pm 2.0 \mathrm{~mm} \mathrm{ML}$. The guide cannulae were lowered slowly $(0.5 \mathrm{~mm} / 5 \mathrm{~min})$ to a depth of $-1.9 \mathrm{~mm}$ subdura. The cannulae were cemented in place with dental acrylic. An electronic connector strip with pins for ground and two EMG wires was fitted between the two guide cannulae and grounded to the skull screws. The EMG wires were implanted under the right eyelid and the entire apparatus was cemented in place (Weiss et al. 1999). Rats were given Buprenex $(0.05 \mathrm{mg} / \mathrm{kg})$ post-surgery to alleviate any discomfort.

Cannulae placements were verified histologically after training was completed. Animals were given a lethal dose of barbiturate (0.15 mL i.p.) then transcardially perfused with $0.9 \%$ saline followed by $10 \%$ formalin. After perfusion, the brains were carefully immersed in $10 \%$ formalin for a minimum of $24 \mathrm{~h}$. Eighty micron coronal sections were made with a freezing cryostat, and every second section was kept and mounted on gelatin coated slides. Sections were stained with cresyl violet to reveal cell death or excessive damage surrounding the injection cannulae. Animals with incorrectly placed cannulae or excessive tissue damage were not included in the study. A diagram of the most ventral extent of each cannula is shown in Figure 1A. Paxilline, a BK-channel blocker, is a peptide and there is little known about the motility of this molecule in the brain. From previous in vivo recording work (Matthews et al. 2008), it is known that the BK blocker is active and able to diffuse at least a radius of $1.7 \mathrm{~mm}$ from the tip of the cannula. To approximate the spread of paxilline in the present study, $1.0 \%$ ibotenic acid was injected into two animals at the completion of training in a manner that exactly mimicked the paxilline injections in volume and rate. Five days after excitotoxic lesion, animals were sacrificed and their brains processed. Figure 1B shows the maximum and minimum extent of cell loss due to ibotenic acid lesion.

Freely moving animals were injected with vehicle (1\% DMSO in saline), drug (1 $\mu \mathrm{M}$ paxilline in saline), or nothing 20-30 min 

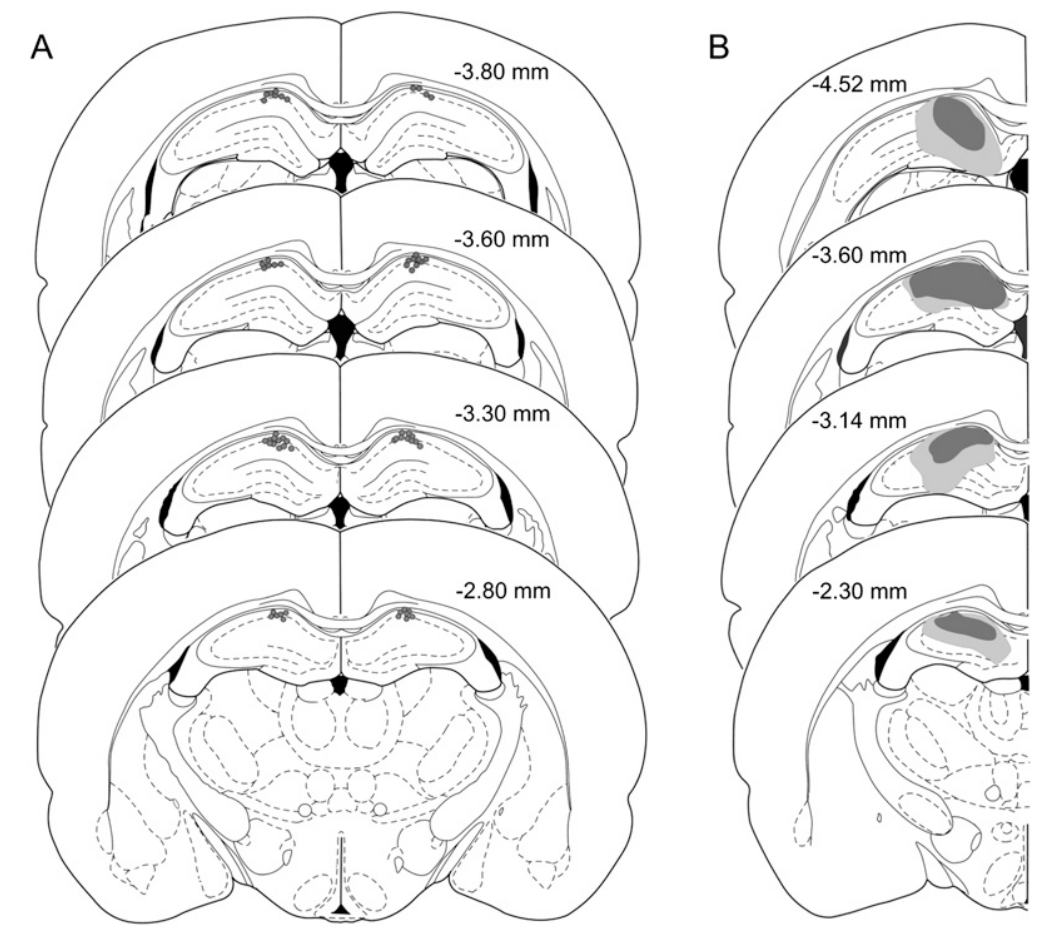

Figure 1. Cannula placement and drug spread. (A) Cannulae were bilaterally implanted to terminate directly above the CA1 layer of the hippocampus. Placement was verified after the behavioral experiments. Gray dots indicate the location of the tip of each cannula for animals included in the study. (B) The spread of the drug was approximated by injecting $1.0 \%$ ibotenic acid into the left hemisphere of two animals at the end of training. The injection volume and rate were the same as those used for the study. The right hemisphere served as a within-animal control for the action of the ibotenic acid. The maximal (light gray) and minimal (dark gray) spread are shown. Measurements are relative to bregma. (Adapted from Paxinos and Watson [1998] and reprinted with permission from Elsevier (c)1998.)

before the start of training, including the first habituation session. The trainer was blind to the identity of the injected substance. Infusions were performed using two $2 \mu \mathrm{L}$ Hamilton syringes connected by lengths of flexible, oil filled tubing to 33 gauge infusion needles, which extended $0.5 \mathrm{~mm}$ beyond the end of the guide cannulae. One microliter of sterile solution was infused into each hemisphere at a constant rate of $0.2 \mu \mathrm{L} / \mathrm{min}$ using a Stoelting double-barrel infusion pump. The injection needles were left in place for $1 \mathrm{~min}$ following the injection to allow diffusion away from the tip of the needle.

Trace eyeblink conditioning is an associative learning task in which a neutral conditioned stimulus (CS) is paired with an unconditioned stimulus (US) that elicits a reflexive eyelid closure. The insertion of a stimulus-free "trace" interval between the CS and the US makes this task strongly dependent on the hippocampus (Solomon et al. 1986; Moyer Jr. et al. 1990). After repeated pairings of the CS and the US, if an association has been learned, the subject will begin to blink during the trace period in anticipation of the US. Trace eyeblink conditioning procedures as described by Weiss et al. (1999) were followed. Training sessions were conducted in a sound-attenuating chamber and controlled with a custom-designed LabVIEW (National Instruments) program; eyelid EMG data were integrated online during training. Animals were connected to the recording computer via the implanted connector strip; a short tether served the dual purpose of allowing EMG activity to be monitored and positioning an air puff delivery tube in front of the eye, while the rat was freely moving. On the first day, the subjects received a session of stimulus-free habituation to the training chamber lasting as long as a conditioning session. The subsequent $5 \mathrm{~d}$ were training sessions. Conditioned animals received 30 trials per session ( $30 \mathrm{~s}$ average ITI) for a total of 150 CS-US pairings, consisting of a tone stimulus (CS, $80 \mathrm{~dB}, 250 \mathrm{~ms}$ ) paired with a corneal air puff (US, 3-5 psi, $100 \mathrm{~ms}$ ) with a $250 \mathrm{~ms}$ stimulus-free trace interval interposed.

The primary measure of $\mathrm{tEBC}$ learning used in this study was a correctly timed eyelid closure, i.e., an eyelid closure that begins during the trace interval and continues until the air puff. Eyelid activity was measured with an implanted EMG electrode. The division of eyelid responses into "adaptive" and "nonadaptive" categories has been used in other studies (Garcia et al. 1999). For this reason, we analyzed eyelid closure during the entire tone/trace period and during only the last $200 \mathrm{~ms}$ preceding the air puff. Responses given during the $200 \mathrm{~ms}$ preceding the US are termed adaptive conditioned responses (CR). Figure 2 shows the timing of the tone, trace, and air puff; the timeline for each type of response; and an integrated EMG. Any trials in which the baseline activity in the $500 \mathrm{~ms}$ preceding CS presentation exceeded two standard deviations were discarded. Eye closure was defined as greater than four standard deviations above baseline. Averages for all relevant measures for each session were compared between groups for training sessions $1-5$ using repeated-measures ANOVA. Learning across training sessions within each group was assessed with a planned comparison ANOVA using StatVIEW software. The stimulus-free habituation session was

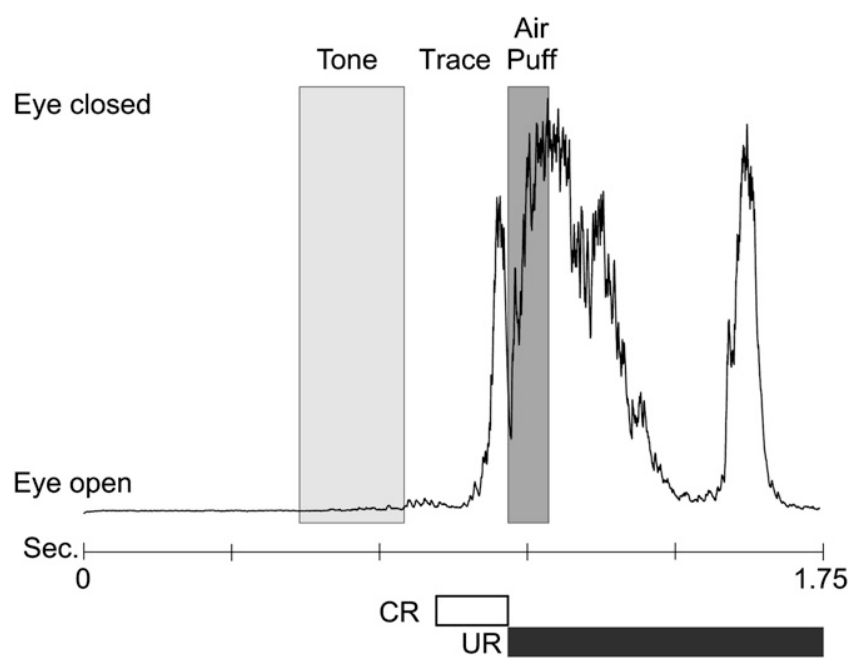

Figure 2. Timing of conditioned responses (CRs) in relation to the tone, trace, and air puff. The integrated EMG shows a robust adaptive eyelid closure in anticipation of the air puff. The tone lasted $250 \mathrm{~ms}$ and was followed by a $250 \mathrm{~ms}$ trace period. The air puff was $100 \mathrm{~ms}$ long. Below the EMG trace is a time line showing the period for an adaptive CR and an unconditioned response (UR). 
excluded from all between-group analyses. The percentage of aCRs during habituation is shown in Figure 3 to provide the baseline level of spontaneous eyelid closures.

The concentration of paxilline was selected based on the dose that achieved a maximal increase in spontaneous firing in vivo (Matthews et al. 2008). Two groups were designed to control for pressure effects of the injection or the stress and tissue damage of the cannulation surgery. These were a vehicle group (1\% DMSO in saline) and a sham group (empty needles). In addition, the learning behavior of these cannulated animals was compared to a separate group of animals that had only the EBC head apparatus implanted (non-cannulated).

Rats that received an infusion of paxilline $(1 \mu \mathrm{M})$ immediately preceding training were significantly slower to acquire the task as measured by the percent of adaptive CRs exhibited across all training sessions $\left(F_{(3,26)}=3.155, P=0.042\right.$, repeated-measures ANOVA, Fisher's PLSD, $P<0.02$ ) (Fig. 3). The percentage of responses during the entire tone/trace period showed a trend toward reduced learning in the drug group $\left(F_{(3,26)}=2.729, P=\right.$ 0.064 , repeated-measures ANOVA).

Other parameters of the eyelid closure were examined. There were no significant differences in the peak, onset, duration, or area of the adaptive CR. There was also no difference between groups in the onset of the air-puff elicited eyelid closure (unconditioned response [UR]), suggesting that the drug did not cause decreased sensitivity to the US. Finally, the baseline eyelid EMG activity of all four groups during the stimulus-free habituation session was not significantly different, suggesting that the drug did not suppress or enhance spontaneous eyelid activity.

All animals in the study showed improved performance across the training sessions $\left(F_{(4,104)}=21.810, P<0.0001\right.$, repeated-measures ANOVA). Further analyses revealed that animals in the drug group also showed continuous learning over the training days (Session 1: $16.1 \% \pm 4.9 \%$, Session 5: $56.8 \% \pm$ $\left.8.2 \% ; F_{(4,32)}=6.961 ; P=0.0004\right)$, and eventually the drug group

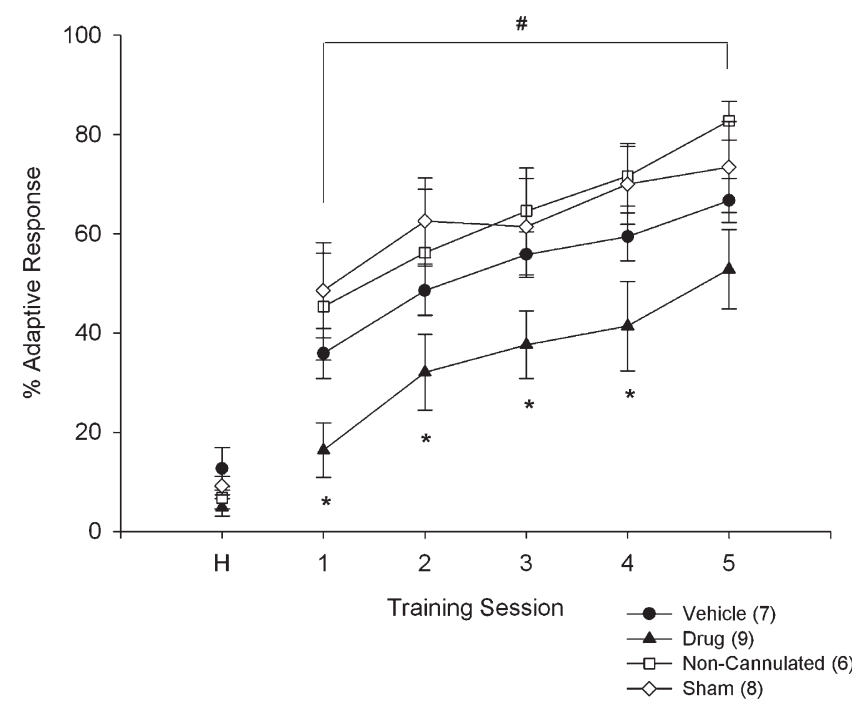

Figure 3. BK block slows learning of trace eyeblink conditioning. Vehicle, drug, and sham animals were injected and immediately trained on the trace eyeblink task over $6 \mathrm{~d}$, one session each day. (Noncannulated animals were trained in two sessions per day for $3 \mathrm{~d}$.) The first session for all groups was stimuli-free habituation. Each session consisted of 30 pairings of a CS tone with a US air puff. Injection of the BK blocker paxilline resulted in slower acquisition of the task $\left({ }^{*} P<0.05\right)$, although animals in all groups were eventually able to learn the task (\#P< 0.001). reached a percentage of adaptive CRs statistically comparable to the controls $\left(F_{(3,26)}=2.377, P=0.093\right.$ for Session 5, ANOVA).

There is a precedent for anticipating enhanced learning after in vivo pharmacological manipulations of intrinsic excitability (Disterhoft and Oh 2006). The long-lasting post-burst AHP is reduced in hippocampal cells from animals that have learned a hippocampus-dependent task (Moyer Jr. et al. 1996; Oh et al. 2003), and increased in aging animals that have difficulty learning. Pharmacologically reducing the post-burst AHP in vivo with calcium channel blockers (nimodipine) (Deyo et al. 1989), acetylcholine agonists (CI-1017) (Weiss et al. 2000), or cholinesterase inhibitors (galantamine and metrifonate) (Kronforst-Collins et al. 1997; Weible et al. 2004) leads to improved learning in aging animals. Thus, the finding that blocking the BK channel results in slowed learning is somewhat surprising, given the previous report of a reduction in the BK-mediated fast AHP after learning tEBC (Matthews et al. 2008). Several explanations may account for why pharmacologically reducing the fast AHP in vivo impaired rather than improved learning.

First, the reduction of the fast AHP seen with channel blockers in in vitro experiments is of greater magnitude than the reductions in the fast AHP seen after learning; additionally, the fast AHP in cells from trained animals can be further reduced in vitro with paxilline or iberiotoxin (Matthews et al. 2008). It could be that there is an important difference between reducing the BKmediated current, as is seen after learning, and completely blocking it using a drug. Reducing the fast AHP increases intrinsic excitability, and completely blocking the BK channel increases excitability even further. However, it is important to emphasize recent research showing how excessive excitability can be detrimental to learning. An early indicator of mild cognitive impairment detected with fMRI is hyperactivity in the hippocampus and medial temporal lobe (Miller et al. 2008). At the cellular level, saturating in vivo hippocampal LTP results in impaired spatial learning due to increased epileptiform activity, rather than saturated synaptic plasticity (McNamara et al. 1993). Finally, work with a knockout model of the $\beta-4$ subunit of the BK channel shows that this calcium- and voltage-dependent channel helps to regulate hyperexcitability and reduce the occurrence of temporal lobe seizures (Brenner et al. 2005). Although intrahippocampal paxilline infusion did not cause epileptiform activity in in vivo recordings, or observable seizure behavior (Juhng et al. 1999), it is possible that the drug caused pathological increases in excitability that impeded learning.

Second, unregulated or meaningless increases in baseline neural activity in the hippocampus increase background noise, effectively decreasing the signal-to-noise ratio for the whole network, making it more difficult to distinguish important, information-bearing activity from background noise. In delay conditioning (where there is no trace interval between the CS and US), ablation of the hippocampus does not disrupt eyeblink conditioning (Solomon et al. 1986; Hesslow 1994), however, increasing (Salafia et al. 1979) or suppressing (Solomon et al. 1983) hippocampal activity has a strong retarding effect on learning this task. Disrupting synaptic transmission in a subregion of the hippocampus also impairs spatial learning (Niewoehner et al. 2007), further illustrating how a disturbance of information processing at a single junction of the trisynaptic circuit can impair learning. BK channels are present in presynaptic terminals, as well as at the soma, where they participate in controlling transmitter release. Blocking BK channels decreases failures and increases the amplitude of EPSCs at CA3-CA3 synapses (Raffaelli et al. 2004). The infusion of paxilline may have also increased the efficacy and frequency of transmitter release at the CA3-CA1 synapse in the present experiment. It may be that by selecting a dose of paxilline that caused a maximum increase in in vivo spontaneous activity, we 
overdosed the hippocampus to levels of excitability that interfered with stimulus processing, thereby impairing and slowing learning.

Finally, the fast AHP is largely mediated by the BK channel, but other potassium currents also play a role in action potential repolarization. The A-type potassium current in particular has been implicated in learning-related excitability changes and is active during an action potential (Giese et al. 1998). Changing action potential repolarization dynamics also alters the calcium influx into cells (Zhou et al. 2005), which can have far reaching effects on other calcium-dependent processes, such as gene regulation, synaptic plasticity, or protein expression. The learning impairment seen in this study might be due to secondary effects on other potassium currents or calcium-dependent processes.

This study indicates that normal activity of BK channels is required for acquisition of the tEBC task. The channel may act to maintain neurons within a narrow window of excitability, keeping neurons within an operating range of "optimal excitability." BK channel activity is strongly modulated by kinase-phosphotase activity (Reinhart et al. 1991; Loane et al. 2006), and reduction of BK-mediated current through modulators of these molecules may have a more beneficial impact on learning. Since blocking of the BK channel with paxilline impedes learning, this drug may not present a useful tool for pharmacological learning-enhancement manipulations.

\section{References}

Brenner, R., Chen, Q.H., Vilaythong, A., Toney, G.M., Noebels, J.L., and Aldrich, R.W. 2005. BK channel $\beta 4$ subunit reduces dentate gyrus excitability and protects against temporal lobe seizures. Nat. Neurosci. 8: 1752-1759.

Deyo, R.A., Straube, K.T., and Disterhoft, J.F. 1989. Nimodipine facilitates associative learning in aging rabbits. Science 243: 809-811.

Disterhoft, J.F. and Oh, M.M. 2006. Learning, aging and intrinsic neuronal plasticity. Trends Neurosci. 29: 587-599.

Garcia, K.S., Steele, P.M., and Mauk, M.D. 1999. Cerebellar cortex lesions prevent acquisition of conditioned eyelid responses. J. Neurosci. 19: 10940-10947.

Giese, K.P., Storm, J.F., Reuter, D., Fedorov, N.B., Shao, L.R., Leicher, T., Pongs, O., and Silva, A.J. 1998. Reduced $\mathrm{K}^{+}$channel inactivation, spike broadening, and after-hyperpolarization in $\mathrm{Kv} \beta 1$ 1.1-deficient mice with impaired learning. Learn. Mem. 5: 257-273.

Hesslow, G. 1994. Inhibition of classically conditioned eyeblink responses by stimulation of the cerebellar cortex in the decerebrate cat. J. Physiol. 476: $245-256$.

Juhng, K.N., Kokate, T.G., Yamaguchi, S., Kim, B.Y., Rogowski, R.S., Blaustein, M.P., and Rogawski, M.A. 1999. Induction of seizures by the potent $\mathrm{K}^{+}$channel-blocking scorpion venom peptide toxins

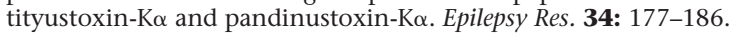

Kronforst-Collins, M.A., Moriearty, P.L., Schmidt, B., and Disterhoft, J.F. 1997. Metrifonate improves associative learning and retention in aging rabbits. Behav. Neurosci. 111: 1031-1040.

Loane, D.J., Hicks, G.A., Perrino, B.A., and Marrion, N.V. 2006. Inhibition of BK channel activity by association with calcineurin in rat brain. Eur. J. Neurosci. 24: 433-441.

Matthews, E., Weible, A.P., Shah, S., and Disterhoft, J.F. 2008. The BKmediated fAHP is modulated by learning a hippocampus-dependent task. Proc. Natl. Acad. Sci. 39: 15154-15159.

McNamara, R.K., Kirkby, R.D., dePape, G.E., Skelton, R.W., and Corcoran, M.E. 1993. Differential effects of kindling and kindled seizures on place learning in the Morris water maze. Hippocampus 3: 149-152.
Miller, S.L., Fenstermacher, E., Bates, J., Blacker, D., Sperling, R.A., and Dickerson, B.C. 2008. Hippocampal activation in adults with mild cognitive impairment predicts subsequent cognitive decline. J. Neurol. Neurosurg. Psychiatr. 79: 630-635.

Moyer Jr., J.R., Deyo, R.A., and Disterhoft, J.F. 1990. Hippocampectomy disrupts trace eyeblink conditioning in rabbits. Behav. Neurosci. 104: 243-252.

Moyer Jr., J.R., Thompson, L.T., and Disterhoft, J.F. 1996. Trace eyeblink conditioning increases CA1 excitability in a transient and learningspecific manner. I. Neurosci. 16: 5536-5546.

Nelson, A.B., Krispel, C.M., Sekirnjak, C., and du Lac, S. 2003. Long-lasting increases in intrinsic excitability triggered by inhibition. Neuron 40: 609-620.

Niewoehner, B., Single, F.N., Hvalby, O., Jensen, V., Meyer zum Alten Borgloh, S.M., Seeburg, P.H., Rawlins, J.N., Sprengel, R., and Bannerman, D.M. 2007. Impaired spatial working memory but spared spatial reference memory following functional loss of NMDA receptors in the dentate gyrus. Eur. J. Neurosci. 25: 837-846.

Oh, M.M., Kuo, A.G., Wu, W.W., Sametsky, E.A., and Disterhoft, J.F. 2003. Water maze learning enhances excitability of CA1 pyramidal neurons. $J$. Neurophysiol. 90: 2171-2179.

Paxinos, G. and Watson, C. 1998. The rat brain, 4th ed. Elsevier, Amsterdam, The Netherlands.

Raffaelli, G., Saviane, C., Mohajerani, M.H., Pedarzani, P., and Cherubini, E. 2004. BK potassium channels control transmitter release at CA3-CA3 synapses in the rat hippocampus. J. Physiol. 557: 147-157.

Reinhart, P.H., Chung, S., Martin, B.L., Brautigan, D.L., and Levitan, I.B. 1991. Modulation of calcium-activated potassium channels from rat brain by protein kinase A and phosphatase 2A. J. Neurosci. 11: 16271635.

Salafia, W.R., Chiaia, N.L., and Ramirez, J.J. 1979. Retardation of rabbit nictitating membrane conditioning by subseizure electrical stimulation of hippocampus. Physiol. Behav. 22: 451-455.

Santini, E., Quirk, G.J., and Porter, J.T. 2008. Fear conditioning and extinction differentially modify the intrinsic excitability of infralimbic neurons. J. Neurosci. 28: 4028-4036.

Simon, B.B., Knuckley, B., and Powell, D.A. 2004. Galantamine facilitates acquisition of a trace-conditioned eyeblink response in healthy, young rabbits. Learn. Mem. 11: 116-122.

Solomon, P.R., Solomon, S.D., Schaaf, E.V., and Perry, H.E. 1983. Altered activity in the hippocampus is more detrimental to classical conditioning than removing the structure. Science 220: 329-331.

Solomon, P.R., Vander Schaaf, E.R., Thompson, R.F., and Weisz, D.J. 1986 Hippocampus and trace conditioning of the rabbit's classically conditioned nictitating membrane response. Behav. Neurosci. 100: 729744 .

Storm, J.F. 1987. Action potential repolarization and a fast afterhyperpolarization in rat hippocampal pyramidal cells. J. Physiol. 385: 733-759.

Weible, A.P., Oh, M.M., Lee, G., and Disterhoft, J.F. 2004. Galantamine facilitates acquisition of hippocampus-dependent trace eyeblink conditioning in aged rabbits. Learn. Mem. 11: 108-115.

Weiss, C., Knuttinen, M.G., Power, J.M., Patel, R.I., O'Connor, M.S., and Disterhoft, J.F. 1999. Trace eyeblink conditioning in the freely moving rat: Optimizing the conditioning parameters. Behav. Neurosci. 113: 1100-1105.

Weiss, C., Preston, A.R., Oh, M.M., Schwarz, R.D., Welty, D., and Disterhoft, J.F. 2000. The M1 muscarinic agonist CI-1017 facilitates trace eyeblink conditioning in aging rabbits and increases the excitability of CA1 pyramidal neurons. J. Neurosci. 20: 783-790.

Zhou, Y.D., Acker, C.D., Netoff, T.I., Sen, K., and White, J.A. 2005. Increasing $\mathrm{Ca}^{2+}$ transients by broadening postsynaptic action potentials enhances timing-dependent synaptic depression. Proc. Natl. Acad. Sci. 102: $19121-19125$.

Received November 24, 2008; accepted in revised form December 10, 2008. 


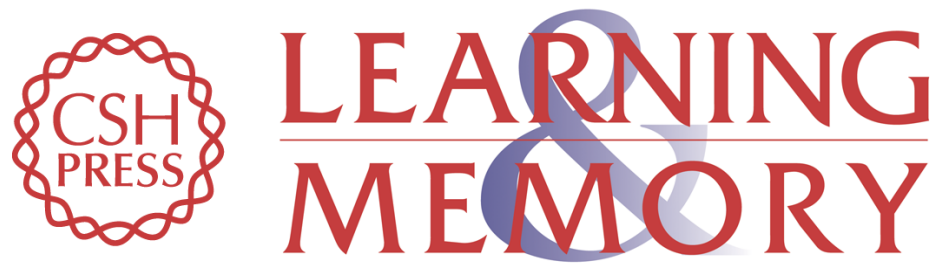

\section{Blocking the BK channel impedes acquisition of trace eyeblink conditioning}

Elizabeth A. Matthews and John F. Disterhoft

Learn. Mem. 2009, 16:

Access the most recent version at doi:10.1101//m.1289809

References This article cites 29 articles, 12 of which can be accessed free at:

http://learnmem.cshlp.org/content/16/2/106.full.htmI\#ref-list-1

License

Email Alerting Receive free email alerts when new articles cite this article - sign up in the box at the Service top right corner of the article or click here. 